\title{
Copolymerization of carbon monoxide and styrene catalyzed by resin-supported palladium polymer
}

\author{
J. Guo*, B. Wang, C. Zhu \\ Department of Chemical Engineering and Technology, Tianjin University, Tianjin 300072, China
}

Received 2 November 2006; accepted in revised form 13 January 2007

\begin{abstract}
Polyketone was prepared by the copolymerization of carbon monoxide (CO) and styrene (ST) catalyzed by $o$-phenylenediamine resin-supported palladium acetate. Effects of each catalytic system component such as 2,2'-bipyridine, 1,4-quinone and $p$-toluene-sulphonate on the copolymerization were investigated. The resin-supported catalyst and the copolymerization product were characterized by infrared spectroscopy (IR), differential scanning calorimetry (DSC), thermogravimetry (TG), X-ray photoelectron spectroscopy (XPS), scanning electron microscopy (SEM). Results indicated that the resin-supported catalyst has excellent catalytic property. Furthermore, partial catalytic activity was maintained after the catalyst was used for five times.
\end{abstract}

Keywords: polymer synthesis, molecular engineering, polyketone, resin, carbon monoxide, styrene

\section{Introduction}

Alkene-carbon monoxide alternating copolymers are of considerable interest from different viewpoints [1]. Copolymerization using carbon monoxide for polymer materials avoids environmental pollution and makes great uses of carbon resources as well. Numerous polymer materials can be derived from polyketone by chemical modification of the ketone on the main chain [2]. Besides, the ketone auxochrome group on the main chain also provides polyketone with excellent photo decomposition property [3], and the carbonyl groups in the polyketone can be chemically modified easily to other classes of functional polymers.

In the 1940s, Reppe et al. [4] first synthesized copolymer of styrene and carbon monoxide by using $\mathrm{KNi}(\mathrm{CN})_{4}$ as catalyst. However, the catalytic activity was relatively low. In the 1970s, Drent et al. [5] from the Holland Shell Laboratory investigated a series of catalyst with high catalytic and low cost, and applied them into the copolymerization of styrene and carbon monoxide. Since then, enormous efforts have been placed on the research of synthesis of polyketone from carbon monoxide and alkene, and more and more kinds of polyketone have been synthesized [6-10]. Currently, in the area of polyketone synthesis, metal catalysts are mostly ruthenium, rhodium, palladium, osmium, yttrium, platinum, etc. Among those metals, palladium has the highest catalytic activity [11]. However, its high cost makes lowering the cost of polyketone synthesis the most important issue.

Due to the high cost of the catalyst, reuse becomes an effective way to reduce the production cost. Here, the cation resin was used to absorb $\mathrm{Pd}^{2+}$ in the waste solution of the copolymerization. The waste solution was also condensed and recycled, which increased the catalytic activity of the copolymerization [12-16]. Due to the similarity of the bidentate nitrogen structure in both of the $o$ phenylenediamine and 2,2'-bipyridine, we applied the micro-balls of $o$-phenylenediamine resin-sup-

*Corresponding author, e-mail: jintang_guo@sina.com

(C) BME-PT and GTE 
ported palladium acetate in the copolymerization, and investigated the catalytic property and reusability of the catalyst.

\section{Experimental}

\subsection{Raw materials}

Palladium acetate (analytical reagent (AR), ACROS Organics, NJ, USA); styrene (AR, Tianjin $1^{\text {st }}$ Chemicals Factory); carbon monoxide (Beijing Hepubei Air Industry Ltd.); $p$-toluene-sulphonic acid (AR, Tianjin Huazhen Special Chemicals Factory); 2,2'-bipyridine (AR, China Medicals Group/Shanghai Chemicals Company); 1,4quinone (Chemical pure, North China Special Chemicals Development Center); benzene and ophenylenediamine (AR, Tianjin Kemiou Chemicals Ltd.); methanol (AR, Tianjin $1^{\text {st }}$ Chemicals Factory); ethanol (Industrial pure, Tianjin University Kewei Company); chloride ball (Tianjin Nankai University Resin Factory).

\subsection{Purification of raw materials}

The analytical reagent styrene was vacuum distilled and stored at low temperature before use. The pure methanol was distilled at normal pressure for further usage. All other materials were used as received.

\subsection{Preparation of the resin-supported palladium catalyst}

$o$-phenylenediamine resin was obtained by immersing the chloride ball and o-phenylenediamine in the ethanol for 24 hours. The above obtained $o$ phenylenediamine resin was then immersed in benzene with the palladium acetate, stirred at room temperature, and the $o$-phenylenediamine resinsupported Pd catalyst was obtained.

\subsection{Copolymerization}

The copolymerization of $\mathrm{CO}$ and ST was catalyzed by the resin-supported Pd catalyst Into a $110 \mathrm{ml}$ electromagnetic stirring steeliness autoclave, 2,2'bipyridine, 1,4-quinone, $p$-toluene-sulphonic acid, and balls of the resin-supported Pd were added to form the catalytic system. Methanol and styrene were then added, and $\mathrm{CO}$ flowed in at $2 \mathrm{MPa}$. The reaction was kept in a $60^{\circ} \mathrm{C}$ water bath and constant pressure for $2 \mathrm{hrs}$. When the copolymerization is over, the autoclave was cooled down to room temperature and the unreacted $\mathrm{CO}$ was released. Ethanol was used to precipitate the product. The white powder was obtained after washing, filtration, and drying.

\subsection{Characterization of the catalyst and copolymer}

The palladium ion content in the resin was determined by ICP-9000(N+M) (Inductively-coupled plasma emission spectrometer). The infrared spectrum of the aminated resin and the copolymer were measure by the Bio-Rad FTS 3000 spectrometer in the form of pellets pressed with $\mathrm{KBr}$. Differential scanning calorimetry (DSC) analysis of the polyketone was carried out with a Shimadzu DSC-50 in nitrogen atmosphere $(10 \mathrm{ml} / \mathrm{min})$ at the heating rate of $10^{\circ} \mathrm{C} / \mathrm{min}$. The thermal porperties of the copolymer was studied. Thermogravimetric analysis (TGA) of the polyketone was performed using a Shimadzu TGA-50 under the same conditions as thhose of the DSC analysis. The thermal decomposition property of the copolymer was studied by TGA. X-ray photoelectron spectroscopy (XPS) measurements were used to investigate the surface elements and relative contents of the resin-supported catalyst and the copolymer. It was performed on a Perkin Elmer PHI-1600 spectrometer with $\mathrm{Mg} \mathrm{K} \alpha(1253.6 \mathrm{eV})$ radiation and a hemispherical analyzer operating at constant pass energy of $23.5 \mathrm{eV}$. Spectra were recorded with the $\mathrm{X}$-ray generator operated at $3000 \mathrm{~W}$ and the chamber vacuum was $667 \mathrm{nPa}$. The radiation area was $0.8 \mathrm{~mm}^{2}$. $\mathrm{Al} 2 p(74.00 \mathrm{eV})$ in $\mathrm{Al}_{2} \mathrm{O}_{3}$ was used as an internal reference.

\section{Results and discussions}

\subsection{Characterization of the resin-supported catalyst and the copolymer}

Small, brown coloredn aminated resin balls were prepared by the reaction of $o$-phenylenediamine and chloride ball in the ethanol solvent. Reaction of the aminated resin balls with palladium acetate in benzene solvent at room temperature for $24 \mathrm{hrs}$ yielded the resin-supported palladium catalyst. Figure 1 shows above described reactions. 


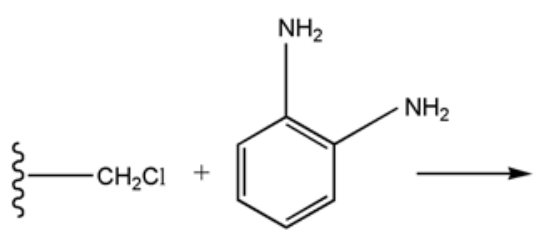<smiles>Nc1ccccc1NCCS</smiles><smiles>CCOC(C)OCCS</smiles>

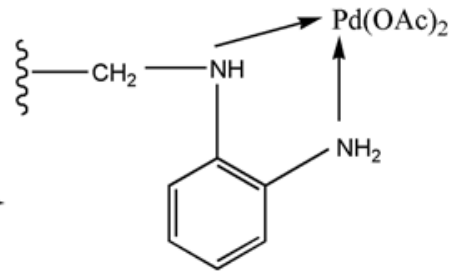

Figure 1. Synthesis route of the resin-supported catalyst

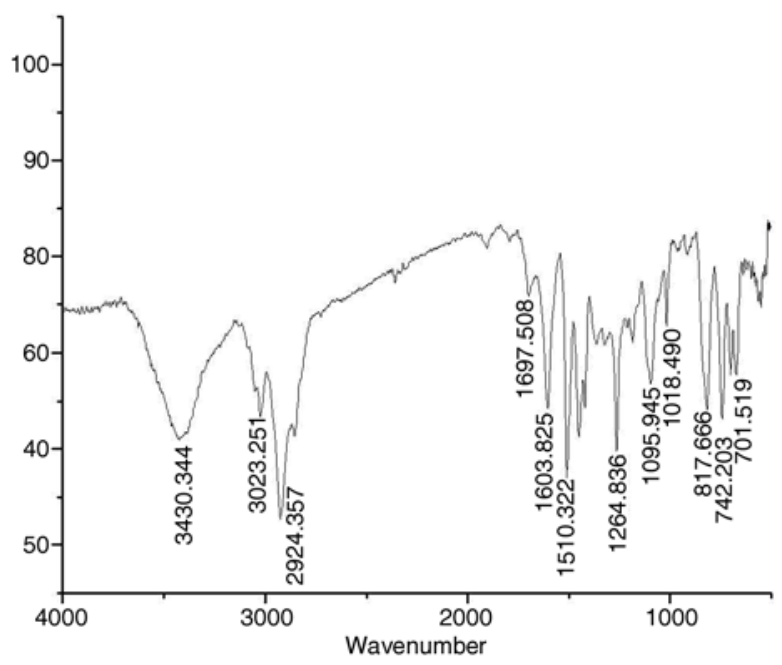

Figure 2. IR of the aminated functional resin

Figure 2 shows the IR spectrum of the aminated resin. Due to the similarity of the characteristic peaks of primary and secondary amines above $3000 \mathrm{~cm}^{-1}$, a broad peak at $3430 \mathrm{~cm}^{-1}$ is observed in the spectrum, which is the result of the overlap of primary and secondary amine characteristic peaks. The peak at $1603 \mathrm{~cm}^{-1}$ is the bending vibration peak of primary amine; the absorption peak at $1018 \mathrm{~cm}^{-1}$ is the stretching vibration peak of $\mathrm{C}-\mathrm{N}$; the strong spectrum area at $1510 \mathrm{~cm}^{-1}$ is the overlap result of $\mathrm{NH}_{3}^{+}$and the benzene skeletons vibration absorption band. Absorption peaks at $697 \mathrm{~cm}^{-1}$ and $750 \mathrm{~cm}^{-1}$ are the in-plane bending vibration peaks of $=\mathrm{CH}$ on the benzene. This is the characteristic absorption band of singly substituted benzene. There are several absorption peaks from $1453 \mathrm{~cm}^{-1}$ to $1600 \mathrm{~cm}^{-1}$, which are caused by the vibration of the benzene skeleton. These are the characteristic absorption bands of the benzene ring. The IR spectrum result revealed that $-\mathrm{Cl}$ in the chloride ball

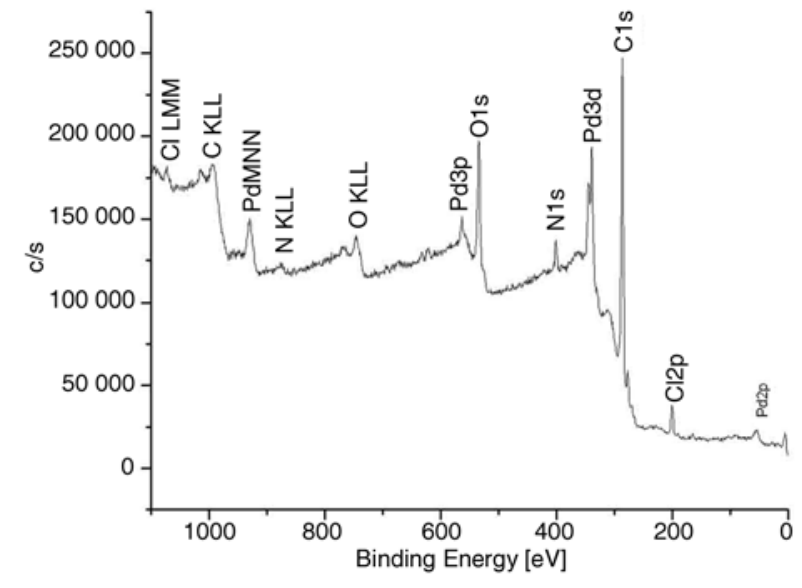

Figure 3. XPS of the resin-supported catalyst

was partly substituted by $-\mathrm{NH}_{3}$ of o-phenylenediamine, and corresponding aminated resin was yielded.

Figure 3 shows that the XPS found the existence of palladium and oxygen, which indicated that the palladium acetate was loaded on the resin surface. A tiny amount of chloride was detected, which possibly origins from unreacted chloromethyl. At the same time, XPS and SEM (Table 1) were applied to measure the palladium content in both the whole body and the core of the resin ball. Results showed $2.1 \mathrm{wt} \%$ and $0.62 \mathrm{wt} \% \mathrm{Pd}$ contents, respectively, which quantitatively proved the existence of the $\mathrm{Pd}$ at the resin surface.

Table 1. Elements on the surface of resin-supported catalyst by SEM

\begin{tabular}{|c|c|}
\hline element & wt\% \\
\hline C K & 91.07 \\
\hline N K & Pd L \\
\hline 8.31 & 0.62 \\
\hline gross & 100.0 \\
\hline
\end{tabular}




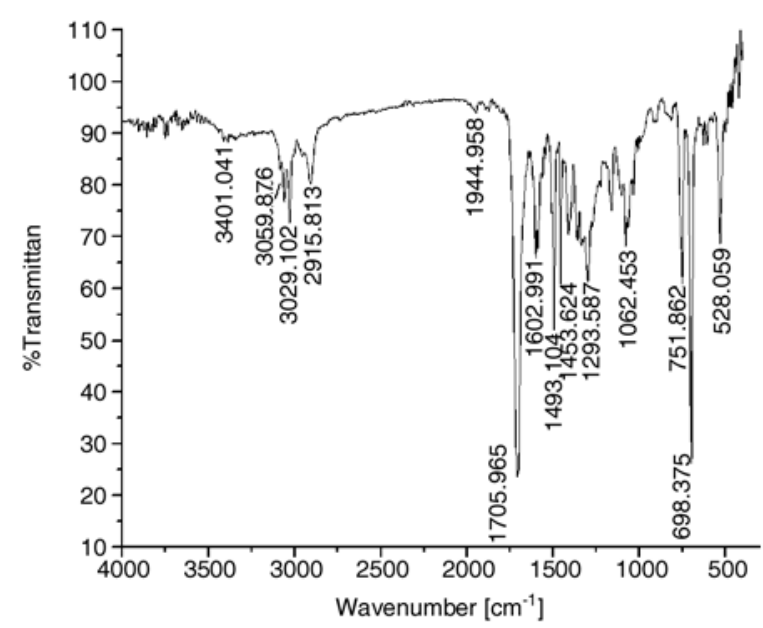

Figure 4. IR of polyketone

White powder was yielded from the copolymerization of CO and ST catalyzed by the resin-supported Pd. Its IR spectrum is shown in Figure 4. It is shown that the product has a strong characteristic absorption peak of ketone at $1703 \mathrm{~cm}^{-1}$. Besides, absorption peaks at $697 \mathrm{~cm}^{-1}$ and $750 \mathrm{~cm}^{-1}$ were induced by the out-of-plane bending vibration of $=\mathrm{CH}$ on the benzene. It is the benzene single substitution characteristic absorption band. There are also several absorption peaks from $1453 \mathrm{~cm}^{-1}$ to $1600 \mathrm{~cm}^{-1}$, which were caused by the vibration of the benzene skeleton and it is the benzene characteristic absorption band. All of the above indicated that the copolymer has structure comes from both the carbon monoxide and the styrene.

Figure 5 shows the Differential scanning calorimetric curve of the copolymerization product which was heated at $10^{\circ} \mathrm{C} / \mathrm{min}$ in the nitrogen atmosphere. Its glass temperature $\left(T_{g}\right)$ is $235^{\circ} \mathrm{C}$ and the melting point is $275^{\circ} \mathrm{C}$.

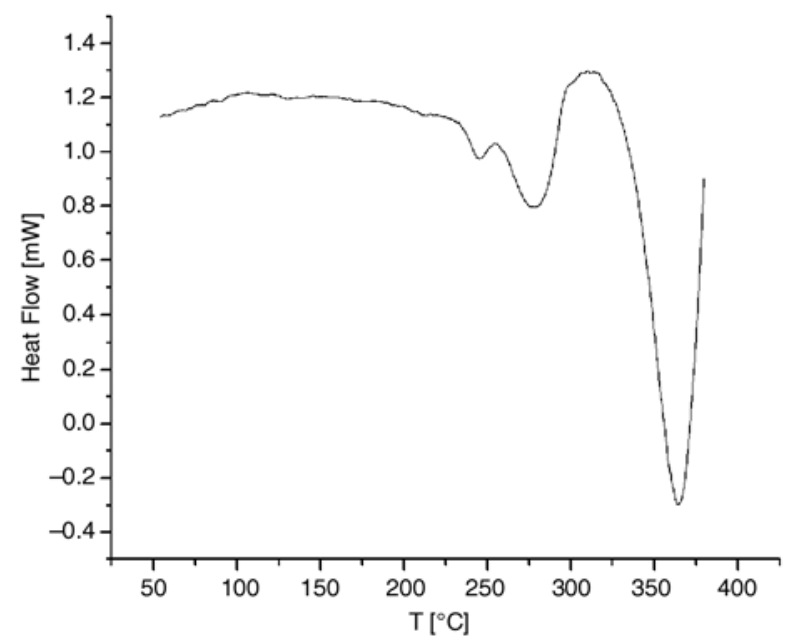

Figure 5. DSC curve of the polyketone

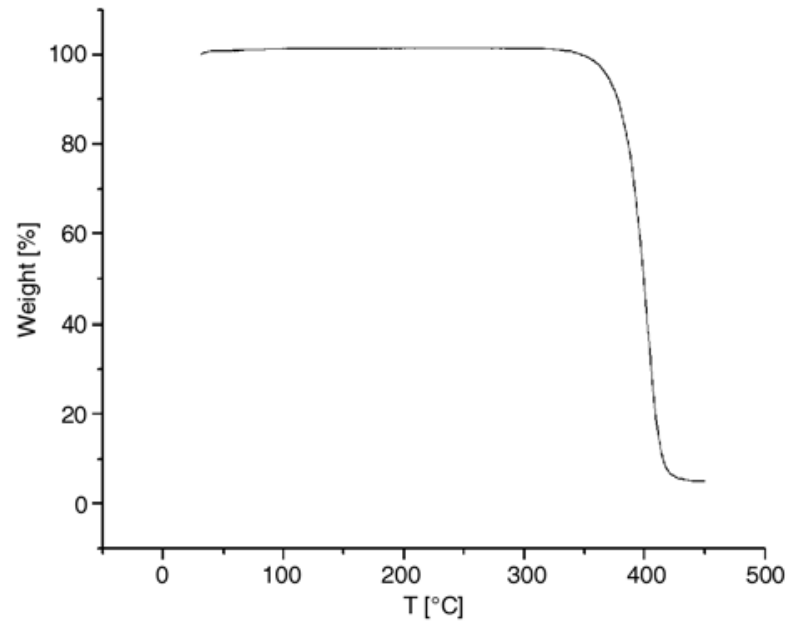

Figure 6. TGA curve of the polyketone

Figure 6 shows the thermogravimetric analysis of the polyketone which was heated at $10^{\circ} \mathrm{C} / \mathrm{min}$ in the nitrogen atmosphere. The initial weight loss temperature is $340^{\circ} \mathrm{C}$ and the complete weight loss temperature is $420^{\circ} \mathrm{C}$.

\subsection{Effect of the resin amount on the yield and the catalytic activity}

Figure 7 shows the effect of the resin amount on the copolymerization yield. It shows that the resin amount has significant affect on the yield. As the resin amount gradually increases, the yield first increases and then decreases. The maximum yield of $250 \mathrm{mg}$ was obtained when the resin amount was $0.3 \mathrm{~g}$. Compared to the traditional homogeneous catalytic system, the active center of the resin-supported catalyst in the reaction system is randomly

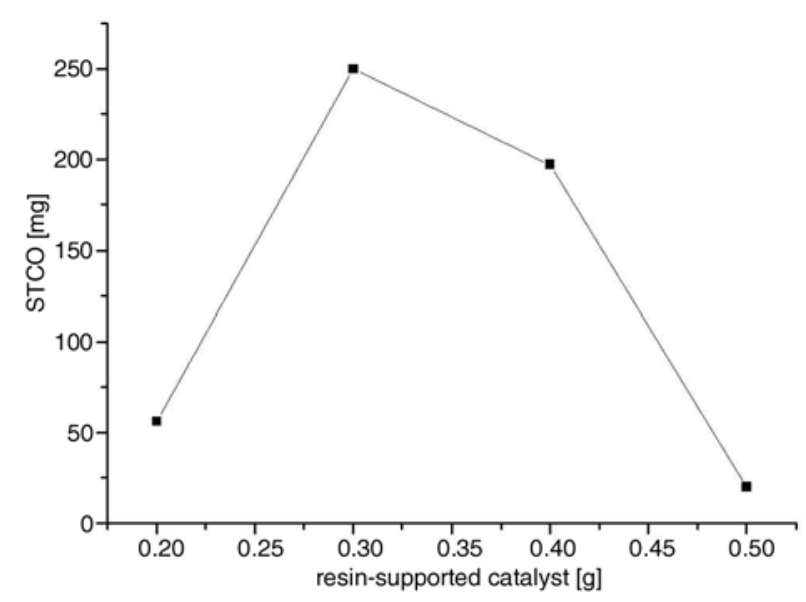

Figure 7. Effect of the amount of the resin-supported catalyst on the yield. Reaction conditions: 2,2'-bipyridine: $1.5 \cdot 10^{-4} \mathrm{~mol}$; 1 ,4-quinone: $20 \cdot 10^{-4} \mathrm{~mol}$; p-toluene-sulphonic acid: $1.5 \cdot 10^{-4} \mathrm{~mol}$; styrene: $15 \mathrm{ml}$; methanol: $6 \mathrm{ml}$; CO: $2 \mathrm{MPa} ; 60^{\circ} \mathrm{C} ; 2 \mathrm{~h}$ 
distributed. When small amount of the resin is used, the effective active center gathered at the resin surface, which results in the absence of the catalytic active center in most reaction area and therefore the yield is low. As the resin amount increases, the resin volume in the autoclave increases. Since the stir is electromagnetic, with the increase of the solid content in the autoclave, the resin was hardly stirred adequately and thus a homogeneous and effective active center can barely be formed. The non-homogeneous distribution of the active center in the reaction system reduces the collision probability of $\mathrm{CO}$ and ST. It therefore reduces the catalytic activity of the reaction. The chloride ball resin used in the experiment is microreticular resin. SEM measurement showed the existence of $\mathrm{Pd}$ in the canal, which increases the difficulty of the catalytic reaction. If the catalytic active center is located in the resin canal, the collision probability of the $\mathrm{CO}$ will be dramatically decreased. The alternating copolymerization can hardly be fulfilled and the catalyst activation energy in the canal is extremely low.

\subsection{Effect of the 2,2-bipyridine amount on the catalytic activity}

In the copolymerization of the $\mathrm{CO}$ with ST catalyzed by $\mathrm{Pd}$, the chelating structure of nitrogen ligand with palladium acetate showed excellent catalytic performance. This is one of reasons why the aminated resin was selected to support the palladium acetate. The kind and the amount of the bidentate ligand have considerable effect on the copolymerization, and also directly affect the stability and activity of the catalytic active center [5, 12]. Hence, 2,2'-bipyridine, which has the best chelating effect with Pd, was selected as the bidentate ligand in the catalytic system. The effect of the 2,2'-bipyridine amount on the polyketone yield was studied and the result is shown in Figure 6.

In Figure 8, it is shown that trivial amount of polyketone was yielded without the ligand addition. It indicates that the $o$-phenylenediamine structure plays the role of the ligand in addition to fixing $\mathrm{Pd}^{2+}$. This assisted the catalytic role of $\mathrm{Pd}$ and resulted in small amount of yield. However, the above ligand structure is an insufficient substitution of the bipyridine ligand structure formed with Pd. When small amount of 2,2'-bipyridine was introduced to the system, the yield was dramatically

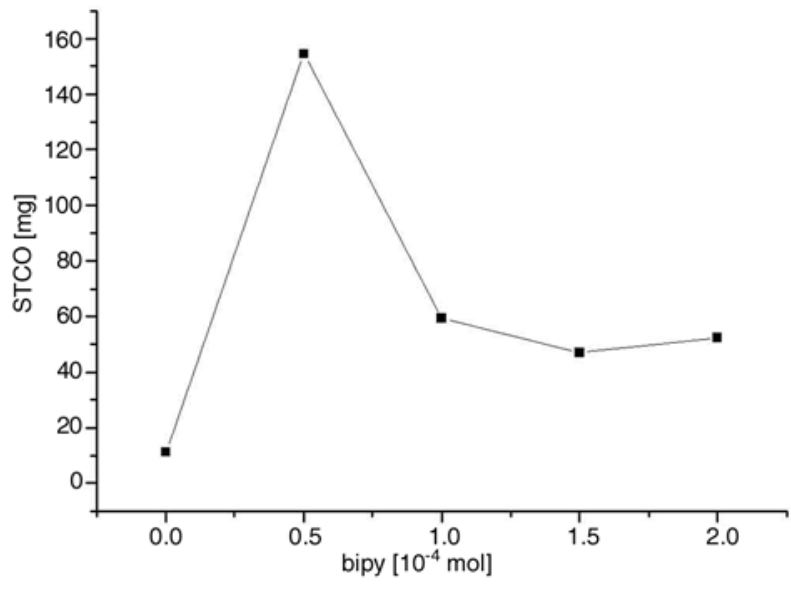

Figure 8. Effect of 2,2'-bipyridine amount on the yield. Reaction conditions were the same as in Figure 7 except that $0.3 \mathrm{~g}$ resin was used and the $2,2^{\prime}$ '-bipyridine amount was varied

increased, which indicated a more stable monochelating $\mathrm{Pd}-\mathrm{N}$ structure was formed and the catalytic performance was also improved. As the ligand amount continued to increase, excess ligands chelated with Pd or gathered around Pd, which resulted in the formation of the bichelating structure. Though the active center is stable, inducing of the monomer became difficult and the yield decreased. The maximum yield of $154.6 \mathrm{mg}$ was obtained when the ligand amount was $0.5 \cdot 10^{-4} \mathrm{~mol}$.

\subsection{Effect of the 1,4-quinone amount on the catalytic activity}

In the copolymerization of the $\mathrm{CO}$ and $\mathrm{ST}, 1,4-$ quinone addition has the following effects: first of all, it acts as an effective anti-copolymerization agent, which prevents the copolymerization of the styrene; secondly, it is also used as a strong oxidizer, which prevents the reduction of the $\mathrm{Pd}^{2+}$. $\mathrm{Pd}(\mathrm{II})-\mathrm{H}$ can be effectively oxidized to $\mathrm{Pd}(\mathrm{II})-\mathrm{OMe}$ and becomes the catalytic active center. Hence, opportune amount of the 1,4-quinone improves the reaction catalytic activity.

Figure 9 shows the effect of the 1,4-quinone amount on the catalytic activity. When trivial amount of the 1,4-quinone was applied, the catalyst is prone to decomposition and loses its catalytic activity. The yield was low. When excess amount of the 1,4-quinone was used, the product was white powder but the yield was low. This is due to the insoluble part of the 1,4-quinone, which makes the system non-homogenous and affects the catalytic activity. The maximum yield of $158.8 \mathrm{mg}$ was 


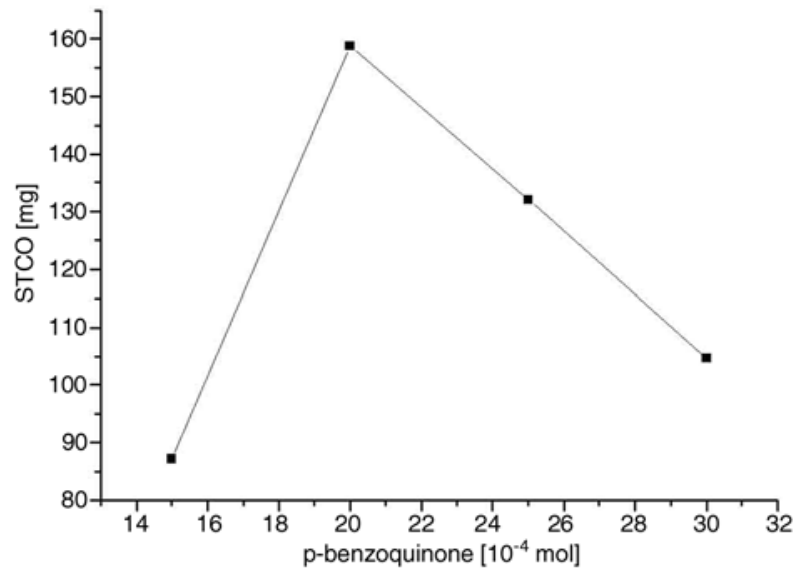

Figure 9. Effect of 1,4-quinone amount on the yield. Reaction conditions were the same as in Figure 7 except that $0.3 \mathrm{~g}$ resin was used and the 1,4quinone amount was varied

obtained when the amount of 1,4-quinone was $20 \cdot 10^{-4} \mathrm{~mol}$.

\subsection{Effect of reused times on the catalytic activity}

Reusability is an important factor in evaluating the performance of the resin-supported catalyst. $0.3 \mathrm{~g}$ resin-supported catalyst was used in the study of its reusability. The effect of the number of reuse on the polyketone yield is shown in Figure 10.

In Figure 10, it is shown that the yield decreased as the number of reuse increases. This indicates the loss of $\mathrm{Pd}$ in the resin-supported catalyst. The loss of the catalyst is unavoidable in the application of the resin-supported catalyst. Therefore, the rate of the catalyst loss is a characteristic property of the resin-supported catalyst.

To better understand the $\mathrm{Pd}$ loss rate in the resinsupported catalyst, the Pd content in an unused,

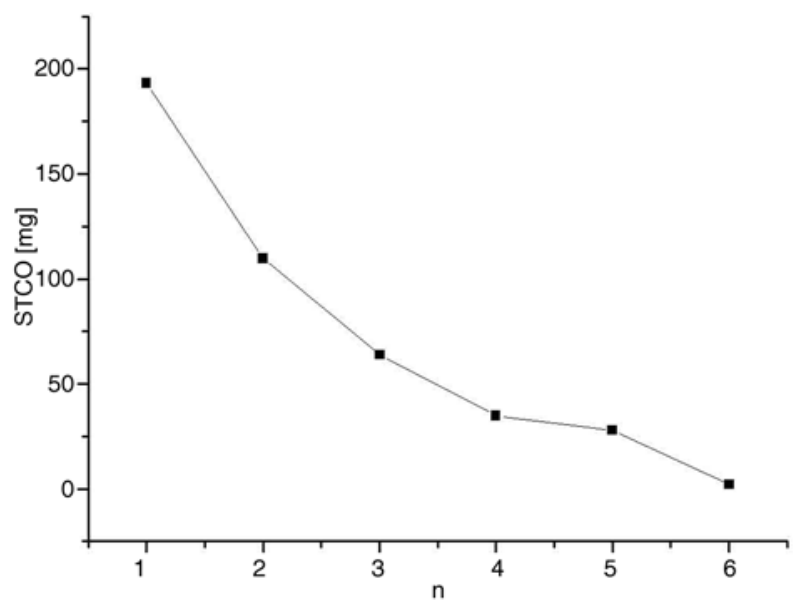

Figure 10. Effect of reused time on the polyketone yield
Table 2. Pd contents in the resin-supported catalyst and the corresponding yield at different numbers of reuse times

\begin{tabular}{|c|c|c|c|}
\hline $\begin{array}{c}\text { reused } \\
\text { number }\end{array}$ & element & result & $\begin{array}{c}\text { polyketone } \\
\text { yield [mg] }\end{array}$ \\
\hline $0 \#$ & $\mathrm{Pd}$ & $2.10 \%$ & 193.2 \\
\hline $1 \#$ & $\mathrm{Pd}$ & $1.06 \%$ & 109.9 \\
\hline $4 \#$ & $\mathrm{Pd}$ & $0.15 \%$ & 27.7 \\
\hline
\end{tabular}

used once, and used four times resins were measured. Results and corresponding polyketone yields are shown in Table 2.

Table 2 shows that the polyketone yield decreases as the Pd reused number increases. 50\% Pd was lost after the first use of the resin; $90 \%$ or above Pd was lost after the resin had been used for four times. The resin-supported catalyst has less and less catalytic activity. The main factor that affects the loss of catalyst is the chelating ability of Pd with $\mathrm{N}$. The chelating between $o$-phenylenediamine and $\mathrm{Pd}$ is a weak force. The palladium acetate loaded on the resin is prone to break and this limits the reuse of this resin-supported catalyst. The key to solve this problem is to select a proper amine compound to form a strong chelating structure with Pd.

\subsection{Investigation of the catalytic properties of the dimethylamine resin-supported $\mathbf{P d}$ catalyst}

Different aminated resins show significant difference in chelating $\mathrm{Pd}$. Above experiments also showed that the chelating effect of $o$-phenylenediamine with $\mathrm{Pd}$ is undesirable, where Pd was lost in reactions. There are two methyls, which are electron-donating groups, in the dimethylamine. Their electron-donating effect increases the density of the electron cloud, which assists the formation of a more stable chelating structure. Hence, $o$ phenylenediamine was substituted by dimethylamine in synthesis of the aminated resin and the resin-supported catalyst. All other reaction conditions were maintained.

However, no product was obtained in the polyketone copolymerization using the dimethylamine resin-supported catalyst. Furthermore, the color of the resin turned from yellow to black. This resinsupported catalyst has no catalytic activity in this experiment.

XPS of the dimethylamine resin-supported catalyst before and after the reaction are shown in Figure 11. 


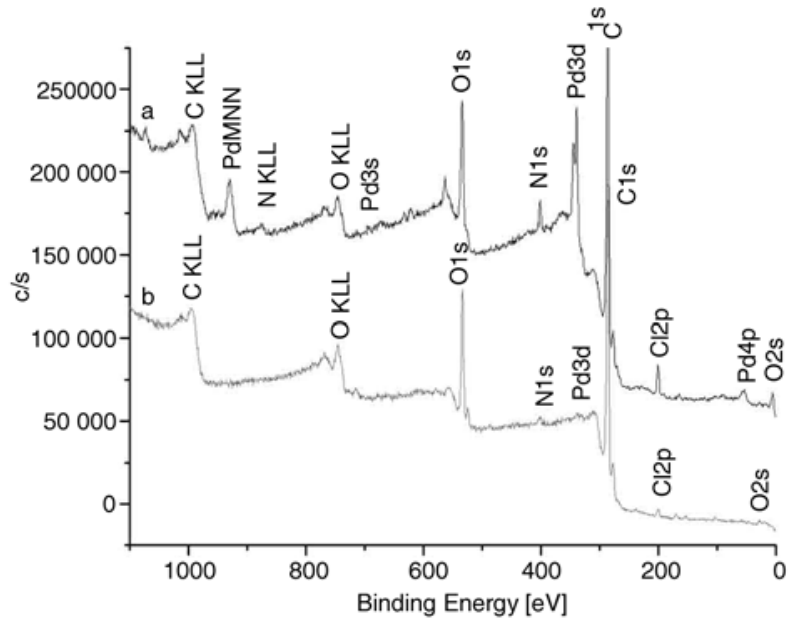

Figure 11. ,XPS of the dimethylamine resin-supported catalyst (a) before use; (b) after use

Comparison of the two XPS results reveals that there are Pd ions loaded on the resin surface before the reaction and small amount of that was left after the reaction. From all of the above results, it is shown that the chelating of $\mathrm{N}$ with $\mathrm{Pd}$ increases due to the electron-donating effect. However, only when the bidentate ligand was used to chelate with the Pd, can its two active sites be fixed and provide the other two active sites for monomer chelating. Dimethylamine only provides one $\mathrm{N}$ to chelate with Pd while the bichelating structure requires two. This is affected by the distribution of the dimethylamine in the resin. When two nitrogen ions are far away from each other, they cannot form chelate with the same Pd. Such structure has no catalytic activity and therefore has no yield. Part of the felled $\mathrm{Pd}^{2+}$ ions was oxidized to black Pd metal.

\section{Conclusions}

$o$-phenylenediamine resin was used to support the Pd catalyst. The catalytic system was composed of 2,2'-bipyridine, 1,4-quinone, and $p$-toluene-sulphonic acid. The alternating polyketone was prepared using $\mathrm{CO}$ and ST with above catalytic system.

Effects of every component in above catalytic system and the reaction time on the catalytic performance were investigated. The maximum yield of $250 \mathrm{mg}$ was obtained with the optimum catalytic system composition of $0.5 \cdot 10^{-4} \mathrm{~mol} 2,2^{\prime}$-bipyridine, $20 \cdot 10^{-4}$ mol 1,4-quinone, and $0.3 \mathrm{~g}$ resin-supported Pd catalyst.

Reusability of the o-phenylenediamine resin-supported Pd catalyst was studies. The catalytic activ- ity of the resin was maintained after five times twohour reactions.

When $o$-phenylenediamine was substituted by dimethylamine, no catalytic activity was showed in the resin-supported Pd catalyst.

\section{Acknowledgements}

This project was financially supported by the National Natural Science Foundation of China (grant no. 20476080).

\section{References}

[1] Drent E., Budzelaar P. H.: Palladium-catalyzed alternating copolymerization of alkenes and carbon monoxide. Chemistry Reviews, 96, 663-681 (1996).

[2] Drent E.: Process for the preparation of polyketones. 0181014 EP (1986).

[3] Jiang Z., Adams S. E., Sen A.: Stereo-and enantioselective alternating copolymerization of $\alpha$-olefins with carbon monoxide synthesis of chiral. Polymers Macromolecules, 27, 2694-2700 (1994).

[4] Reppe W., Magin A.: Production of ketonic bodies. 2562393 U.S. (1951).

[5] Drent E., Broekhoven J., Doyle M. J.: Efficient palladium catalysts for the copolymerization of carbon monoxide with olefins to produce perfectly alternating polyketones. Journal of Organometallic Chemistry, 417, 235-251 (1991).

[6] Luo H. K., Li D. G.: Studies on new palladium(II) catalyst system for copolymerization of $\mathrm{CO}$ with ethylene. Journal of Molecular Catalysis A, 171, 23-31 (2001)

[7] Sen A.: The copolymerization of carbon monoxide. Advances in Polymer Science, 73-74, 125-144 (1986).

[8] Ittel S. D., Johnson L. K., Brookhart M.: Late-metal catalysts for ethylene homo- and copolymerization. Chemical Reviews, 100, 1169-1204 (2000).

[9] Peng J. J.: The copolymerization of carbon monoxide with ethane. Petrochemical Technology, 10, 707-709 (1997).

[10] Barsacchi M., Consiglio G., Medici L., Petrucci G.: Syndiotactic poly(1-oxo-2-phenyltrimethylene):on the mode of the chain growth under palladium catalysis. Angewandte Chemie-International Edition, 30, 989991 (1991).

[11] Desjardins S. Y., Cavell K. J., Hoare J. L., Skelton B. W., Sobolev A. N., White A. H., Keim W.: Single component $\mathrm{N}-\mathrm{O}$ chelated arylnickel(II) complexes as ethene polymerisation and $\mathrm{CO} /$ ethene copolymerisation catalysts. Journal of Organometallic Chemistry, 544, 163-176 (1997).

[12] Wang X. Y., Guo J. T., Xu Y. S., Sun J. W.: Study on the solution copolymerization of carbon monoxide with styrene. Journal of Tianjin University Sience and Technology, 34, 249-252 (2001). 
[13] Feng Y. K., Sun J. W., Zhu Y. B.: Study on the copolymerization of carbon monoxide and styrene by palladium(II)/2,2'-bipyridyl catalyst. Chinese Journal of Catalysis, 17, 50-54 (1996).

[14] Pisano C., Mezzetti A., Consiglio G.: Selectivity of the carbonylation of styrene by cationic palladium complexes. Organometallics, 11, 20-22 (1992).
[15] Guo J. T., Wang X. Y., Liu B.: Effect of acid on the copolymerization of carbon monoxide and styrene. Acta Petrolei Sinica (Petroleum Processing Section), 19, 89-93 (2003).

[16] Guo J. T., Liu B., Li L. Wang X. Y., Sun J. W.: Copolymerization of carbon monoxide and styrene catalyzed by palladium-rare earth catalyst. Chinese Journal of Catalysis, 25, 231-237 (1999). 